\title{
Enhanced model based algorithm to reinforce PV system with dynamic MPPT capability
}

\author{
Md. Ehtesham \\ Department of Electrical and Electronics, Maulana Azad National Urdu University, India
}

\begin{tabular}{l}
\hline \hline Article Info \\
\hline Article history: \\
Received Aug 7, 2020 \\
Revised May 1, 2021 \\
Accepted Jun 18, 2021 \\
\hline
\end{tabular}

\section{Keywords:}

Dynamic

Maximum power point tracking

Model-based algorithm

Partial shading

Photovoltaic

\begin{abstract}
Photovoltaic (PV) power has emerged as the most attractive resource in form of a clean and green energy. However, one major challenge associated with PV interfacing is its intermittent output characteristic which varies dramatically with the operating conditions. Thus designing an effective maximum power point tracking (MPPT) algorithm is a key aspect for optimizing PV system performance. Numerous MPPT algorithms have been proposed earlier having their own specific advantages. However, these are found to have two major limitations which have to be essentially addressed. Firstly, they become ineffective in the dynamic conditions where there is rapid change in environmental parameters like insolation and temperature. Secondly, they fail to discriminate between global and local peaks under partial shading conditions. Therefore, to achieve a reliable and efficient system operation, this paper presents an enhanced model-based (MB) algorithm that overcomes both these deficiencies. Based on new governing equations and precised estimation technique, it predetermines the MPP analytically. First simulated results are obtained where it is tested for dynamic variations of all the three parameters. Then the experimental validation is carried out on a $2 \mathrm{KW}$ installed panel where real time data is recorded through CR1000 data logger and environmental parameters are sensed with elements like pyranometer and humidity sensor. A large number of experimental results are obtained for tracked MPP in the dynamic conditions, which are then summarized in tabular forms. These are finally plotted and compared with simulated results to illustrate the effectiveness of proposed MB algorithm.
\end{abstract}

This is an open access article under the CC BY-SA license.

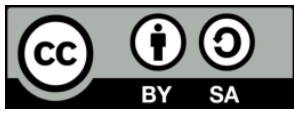

\section{Corresponding Author:}

Md. Ehtesham

Department of Electrical and Electronics

Maulana Azad National Urdu University

Urdu University Road, Telecom Nagar, Gachibowli, Hyderabad, Telangana 500032, India

Email: mdehtesham87@gmail.com

\section{INTRODUCTION}

Over the last few decades there has been a tremendous trend of exploiting the renewable sources of energy on account of various factors like climatic concern, depletion of conventional energy reservoirs, global warming. Among all the renewable sources, photovoltaic (PV) promises to be the most attractive solution as a result of latest advancements in semiconductor materials and power electronic converters. Despite of several advantages that PV system offers [1], one major challenge for the large scale interfacing of PV power is its non-linear output characteristics which vary with the ambient operating conditions [2]. The situation gets further worsened for dynamic conditions where there is rapid variation in environmental conditions [3]. Thus, it demands for comprehensive assessment of characteristics in terms of dynamic 
behaviour and to design advance tracking scheme so that PV panel is essentially made to operate at maximum power point (MPP) in order to optimize the system performance.

There have been a large number of MPPT algorithms applied by the researchers [4]-[8]. These techniques differ from one another from various functional aspects and have their individual pros and cons. Rigorous review of MPPT algorithms reveals that these can be broadly divided into (a) conventional and (b) soft computing techniques. Among the conventional MPPT algorithms, the most preferred methods are perturb and observe (P\&O) [9] and incremental conductance (IC) [10]. Thakran, Singh, Garg, and Mahajan [11] implemented P\&O algorithm where MPPT analysis has been carried out with two different approaches depending on whether perturbations are caused in voltage or duty cycle. It gives an effective tracking approach having biggest advantage in terms of ease of implementation. But the major limitation associated with $\mathrm{P} \& \mathrm{O}$ is that increasing the perturbation rate results into higher oscillations around MPP and hence selecting the step-size remains a dilemma. Kim and Cha [12] proposed a variable step-size approach to overcome this limitation where oscillations at steady state have been reduced significantly and higher system efficiency has been achieved. However, this is capable of tracking MPP only under uniform conditions as it shows sluggish and poor convergence when there is partial shading. Similarly, IC algorithm for MPPT has been effectively implemented by Dhaouadi et al. [13], where system transients and converging response have been analyzed with greater oscillating frequencies. The proposed technique is simple and efficient but the drawback is that it cannot be applied in dynamically changing conditions and also its use is limited to low power application. According to Chauhan et al. [14] overcomes this limitation to some extent where a modified IC algorithm has been proposed by optimizing the controller gain through Grey Wolf technique. However, results are satisfactory only for insolation variation and that too over a small scale only, as dynamic change of other parameters introduces a truncated error. Also, inclusion of optimization algorithm has increased the controller complexity significantly.

With the evolution in intelligence technique, there have been a number of MPPT algorithms developed in recent years on the basis of soft computing. Among these evolutionary techniques, the likes of fuzzy logic control (FLC) [15], artificial neural network (ANN) [16], genetic algorithm (GA) [17], particle swarm optimization (PSO) [18] are found to be explored immensely in this new era of MPPT. According to Nemours and Chowdhury [19], a FLC based MPPT scheme has been implemented which shows good tracking capability for load variations as well. The main advantage of FLC based techniques is the speed of tracking as they can reduce the settling time. However, the major limitation of FLC is that once after defining the rules they cannot be modified and hence large amount of training is required for effective tracking under dynamic insolation and other conditions. Hence FLC is hardly used alone for tracking; rather its combination with other is preferred as hybrid MPPT algorithm [20]. But then of course system complexity becomes a major issue. Abadi et al. [21] proposed a Neuro-Fuzzy based adaptive MPPT algorithm which has improved the conversion efficiency and can be effective for all conditions. But the problem associated with ANN technique is that its computational time and memory requirement is large which significantly increases the complexity. Similarly, GA based algorithms which are mainly employed for stochastic searches, also have the same drawback of large memory and training requirement because of high initial population. PSO is another evolutionary technique which is gaining much of the attention in recent years [22]. It is particularly well suited for tracking of global maxima under partial shading cases where panels are non-uniformly illuminated. However, the affair of parameter initialization is a difficult task in PSO based algorithm which gives rise to the randomness in initial duty cycle. Another drawback of PSO technique is that with the increase in population size, computational difficulty also increases. Here it is important to note that partial shading has several ill effects on PV system performance as it leads to considerable amount of power loss and may even cause damage to panels [23], [24]. Therefore, to design a simple and effective algorithm for minimizing the consequences of partial shading continues to be another challenging aspect in the field of MPPT, even though there have been many proposed work.

Thus in view of detailed analysis of MPPT algorithms as discussed above, it is observed that there exists a number of techniques which perform satisfactorily in uniform conditions with steady variations in environmental parameters. However, there are two major and common drawbacks associated with these methods which need to be addressed for efficient and reliable PV system performance. The first is that they fail to respond effectively when there is dynamic change in the environmental conditions. It means that their operating point drifts away from MPP when the conditions are varied rapidly. Secondly, they show incapability to guarantee the successful tracking of global MPP in case of the partial shading or non-uniform solar irradiance. Motivated by the above deficiencies of existing MPPT techniques, this research work proposes a simplified and enhanced model-based (MB) MPPT algorithm. Here, the distinct feature of proposed MB algorithm is that it works effectively not only in dynamic conditions where insolation or temperature varies rapidly and continuously, rather it has additional capability of tracking the MPP accurately in partial shading conditions also. Based on the new governing equations and precised estimation 
technique, this paper has modified the MB approach to develop an enhanced capability of MPPT in dynamic conditions. Rather than going for heuristic search of MPP as followed by traditional techniques, the proposed MB algorithm utilizes the mathematical modeling and hence pre-determines the MPP analytically. Thus the proposed MB algorithm ensures efficient operation of PV system maintaining stable and reliable supply in the dynamic working conditions.

\section{SYSTEM MODELING FOR PROPOSED MB ALGORITHM}

There are two main aspects involved in accomplishing model based MPPT algorithm which induces the distinguished tracking feature. First of all it implies the task of accurate modeling of PV panel. Several models for the PV panel have been proposed in literature where single diode and two diode model are found to be most common ones [25]-[27]. Single diode model shown in Figure 1 is found to be the most suitable one, offering simplicity with enough accuracy and hence it has been applied here while modeling the PV circuit. A replacement track is created by the parallel connected diode (bypass diode) which prevents the damaging effects of hot-spots. On the basis of equivalent circuit the expression for output current can be simply given as (1).

$$
\mathrm{I}=\mathrm{I}_{\mathrm{ph}}-\mathrm{I}_{\mathrm{D}}-\mathrm{I}_{\mathrm{sh}}
$$

Now, applying theory of semiconductors modified expression can be given as (2).

$$
\mathrm{I}=\mathrm{I}_{\mathrm{ph}}-\mathrm{I}_{\mathrm{o}}\left(\exp \left(\frac{V+R_{S} I}{\alpha V_{t}}\right)-1\right)-\frac{V+R_{S} I}{R_{S h}}
$$

Where, $\mathrm{I}_{\mathrm{o}}$ is the reverse saturation current, $\alpha$ is the ideality factor and $V_{t}$ is the thermal voltage. Here for restricting the panel operation around MPP, characteristic matching is performed by tuning the thermal voltage from time to time with the series resistance $\left(\mathrm{R}_{\mathrm{s}}\right)$. The relevancy of shunt resistance can be ignored as it hardly impacts the behaviour for MPP region. Thus all the factors affecting accuracy of PV model have been taken care and these set a benchmark for governing the PV characteristics under dynamic conditions.

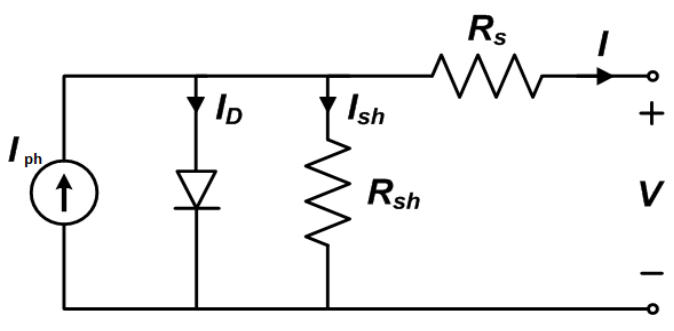

Figure 1. Equivalent single diode PV circuit

Now, the next aspect of MB algorithm which plays a vital role for dynamic tracking is to estimate the parameters precisely. Generally, panel specifications are declared by the manufacturer as given in Table 1 indicating voltage and current ratings for vertices like open circuit, short circuit and MPP. Thus PV model can be defined utilizing rated values from datasheet for Standard Test Conditions (STC: Insolation $\mathrm{S}=1000$ $\mathrm{W} / \mathrm{m}^{2}$, Temperature $\mathrm{T}=25^{\circ} \mathrm{C}$ ). But designing the model using specified data may lead to certain inaccuracy due to dispersion of the manufacturing scheme and unused rated values. Thus to obtain a highly precised model, here in MB algorithm all the environmental parameters are measured precisely with the help of pyranometer and humidity sensor as shown in the next section. Then accordingly PV panel parameters are estimated so as to get a highly accurate tuning with environmental conditions. This predictive approach leads to prediction of error in advance to switching signals fed to converter and thus allows fast jump in the array voltage for rapidly varying environmental conditions. Therefore, rather than going for heuristic search of MPP as followed by common techniques, the proposed MB algorithm utilizes the precised modeling and estimation which enables it to pre-determine the MPP analytically. Consequently, it achieves a significant enhancement in tracking capability under dynamic conditions resulting into high accuracy and efficiency.

Finally for successful implementation of MB algorithm some mathematical operations are carried out which characterizes the PV panel accurately in the region of MPP. Once the operating voltage and current for MPP is obtained then the output power is differentiated and equated to zero as expressed by (3) and (4).

Enhanced model based algorithm to reinforce PV system with dynamic MPPT capability (Md. Ehtesham) 
Imposition of these set of equations leads to fine tuning of panel and bounds the system to operate at its MPP under all sort of dynamic conditions.

$$
\begin{aligned}
& V_{m p}=V_{o c}+V_{T} \ln \left(1-\frac{I_{\mathrm{mp}}}{I_{\mathrm{sc}}}\right)-R_{s} I_{m p} \\
& \frac{d(\mathrm{VI})}{\mathrm{dI}} \mid \mathrm{I}=\mathrm{I}_{\mathrm{mp}}=V_{\mathrm{mp}}+\frac{V_{T} I_{\mathrm{mp}}}{I_{\mathrm{mp}} \mathrm{I}_{\mathrm{sc}}}-R_{s} I_{\mathrm{mp}}=0
\end{aligned}
$$

\section{HARDWARE PLATFORM FOR MB MPPT ANALYSIS}

This section presents the description of hardware setup along with all its components that provided a platform for experimental analysis of MB algorithm. PV panels of $2 \mathrm{KW}$ rating were explored for this work which consists of ten polycrystalline modules, five monocrystalline and five thin film modules each of 100 $\mathrm{W}$ rating. A picture of installed panel is shown by Figure 2 whereas the panel specifications have been summarized in Table 1 as discussed earlier, crux of MB algorithm is the optimum estimation of PV model parameters as a function of their dependency on environmental conditions. Thereby, variations in factors like solar insolation, humidity and wind speed have been tried to be measured with utmost accuracy. Pyranometer has been used here to precisely measure the solar insolation. It is a sensor that measures flux density or global radiation and bears almost a linear relation with the frequency band of total solar radiation. It can also measure the diffuse radiation for which pyranometer is shaded with a disk placed for preventing the direct incidence of beam. Similarly, a humidity sensor has been also used to give the measure of water vapour in the atmosphere. It is related with the temperature in the sense that vapour content of hot air is greater than that of cold air. A picture of installed sensors has been shown in Figure 3.

Table 1. Specifications of practical $2 \mathrm{KW}$ panels installed

\begin{tabular}{ccc} 
S.No. & Parameters (per module) & Value \\
\hline 1 & $\mathrm{P}_{\mathrm{mpp}}$ & $100 \mathrm{~W}$ \\
2 & $\mathrm{~V}_{\mathrm{mpp}}$ & $17.7 \mathrm{~V}$ \\
3 & $\mathrm{I}_{\mathrm{mpp}}$ & $5.7 \mathrm{~A}$ \\
4 & $\mathrm{~V}_{\mathrm{oc}}$ & $21.6 \mathrm{~V}$ \\
5 & $\mathrm{I}_{\mathrm{sc}}$ & $6.3 \mathrm{~A}$ \\
6 & Tolerance & $2 \%$ \\
\hline
\end{tabular}

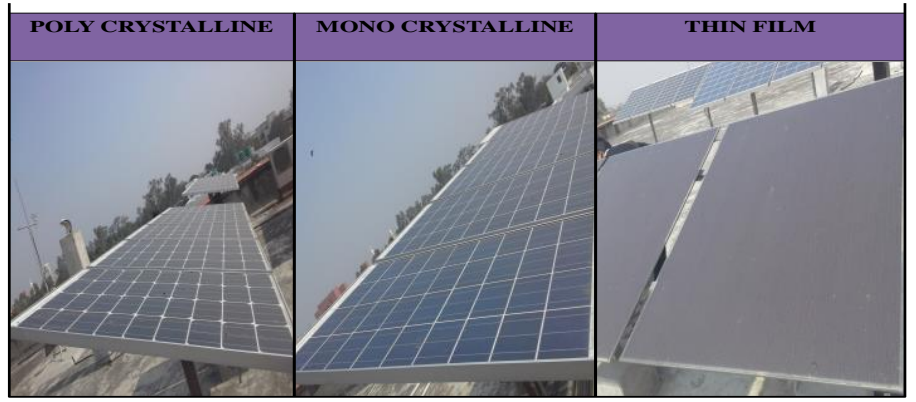

Figure 2. PV panels installed on roof-top

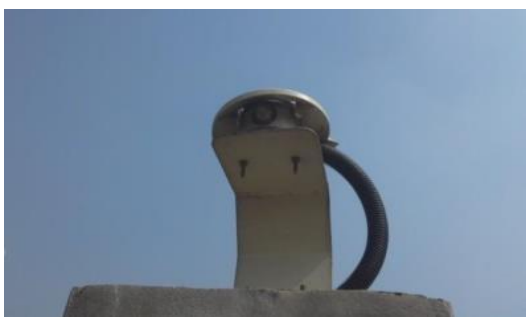

(a)

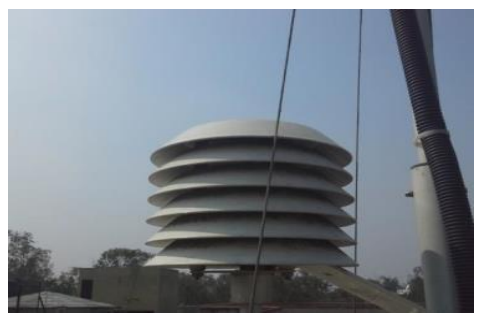

(b)

Figure 3. Installed measuring devices: (a) pyranometer and (b) humidity sensor 
Further, it has to be noted here that any kind of monitoring scheme utilizing sensor monitoring requires a data collection system. For this purpose a portable CR1000 data logger was installed which keeps recording all the measured values of parameters as well as corresponding variations in panel characteristics. This data logger was synchronised with a display board setup inside the lab and at the same time it was also connected to a computer. Hence the instantaneous values of current, voltage and power were available in the display board whereas a continuous record of the same was stored in the system. Figure 4 shows a picture of data logger installed on roof-top and the corresponding display panel is shown in Figure 5.

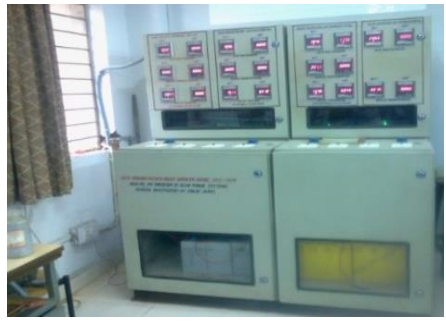

Figure 4. CR 1000 data logger

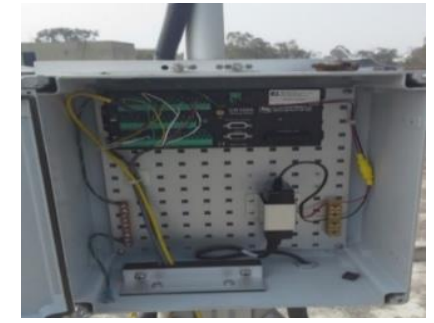

Figure 5. Display board setup

\section{RESULTS AND DISCUSSION}

Having estimated all the parameters precisely with their dependency established on the working conditions, this section now presents the detailed performance analysis of MB algorithm. Here the effectiveness of proposed algorithm is evaluated for rapid variations of all sorts of environmental conditions including partial shading over their full practical range. First the $\mathrm{MB}$ algorithm is tested in MATLAB/Simulink platform where simulated results of tracked MPP for dynamic insolation, temperature and shading are presented. Then the real time performance is analysed where experimental results have been discussed. Finally comparisons of both the results have been carried out to validate the effectiveness of proposed MB algorithm.

\subsection{Simulation results}

Simulink model has been strictly designed on the basis of mathematical equations characterizing the MB algorithm as discussed earlier. First the photocurrent (Iph) is evaluated as a function of solar radiation, then after proper computation of reverse saturation current (Io) and thermal voltage (Vt) diode current is obtained as shown by building blocks in Figure 6. Here it can be seen that diode current has exponential dependency on thermal voltage. Thereafter the summation of diode current and shunt current is subtracted from photocurrent to result into the panel output current as illustrated by the blocks in Figure 7. Thus once the tuned values for current and values are estimated for MPP operation, these are exported to workspace for processing maximum power output as shown in Figure 8.

Now before going for dynamic variations in operating conditions, first of all the characteristic for PV power is analyzed at standard test condition (STC). This sets a benchmark for the rest of evaluation and also ratifies higher tracking efficiency of proposed algorithm. Figure 9 shows the PV curve obtained at STC where it can be observed that MPP is successfully tracked around a voltage of $190 \mathrm{~V}$ with an achieved maximum power of $1920 \mathrm{~W}$. Thus MB algorithm is not only effective for dynamic conditions but it is also capable of yielding a higher tracking efficiency.

Now the response in PV characteristic is tracked for the dynamic working conditions, by rapidly varying different parameters one by one. First the insolation is varied dynamically over the full scale from $100 \mathrm{~W} / \mathrm{m}^{2}$ to $1000 \mathrm{~W} / \mathrm{m}^{2}$ keeping the temperature constant at $25^{\circ} \mathrm{C}$. Therefore, PV characteristics also keep on changing dynamically and corresponding to each characteristic global MPP is tracked effectively. Thus a large number of characteristics are obtained where MPP is tracked separately for dynamic variations of insolation from 100 to 200 to 300 and so on. As it is not possible here to show all the characteristics and MPP tracked for corresponding insolation, hence as a sample value one such characteristic obtained at insolation of $600 \mathrm{~W} / \mathrm{m}^{2}$ is shown in Figure 10. It can be observed here that MPP now occurs around a voltage of $185 \mathrm{~V}$ with tracked maximum power of $1090 \mathrm{~W}$, which is obviously much less than that obtained at STC due to the reduced insolation.

Next, we keep the insolation constant at $1000 \mathrm{~W} / \mathrm{m}^{2}$ and vary the temperature over the scale from 12 ${ }^{\circ} \mathrm{C}$ to $45^{\circ} \mathrm{C}$. Again MPP is successfully tracked with this rapid variation in temperature where global maxima also varies dynamically. One such characteristic obtained at a temperature of $15^{\circ} \mathrm{C}$, is shown in Figure 11 . 
Finally effectiveness of MB algorithm for tracking MPP under the dynamic variations in partial shading is analyzed by calibrating insolation with percentage shading. As shown in Figure 12 MPP is tracked successfully for dynamic increase in shading percentage in steps of $10 \%$. Interestingly, here there is not much variation in the panel voltage till we go beyond $70 \%$ of shading but the current variation is found to be much significant. Even the current becomes negative if shading goes to the limit of $100 \%$ because of reverse saturation impact.

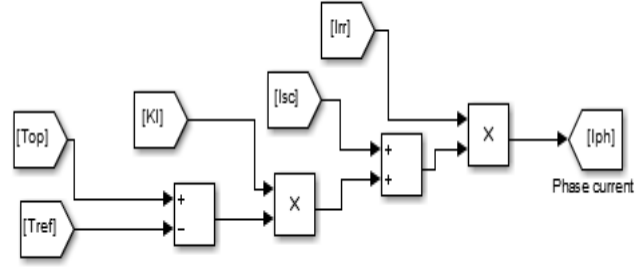

(a)

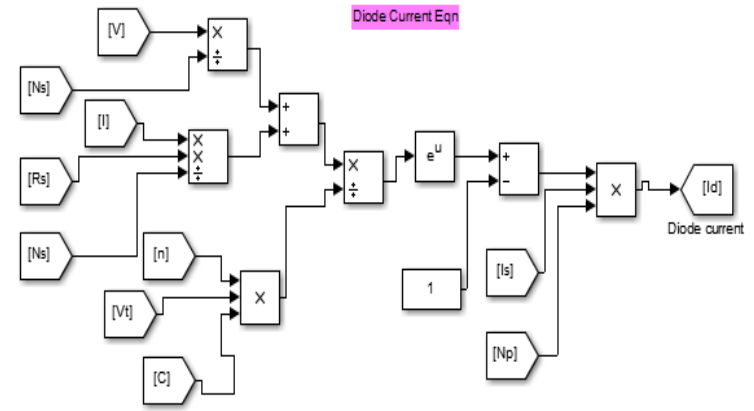

(b)

Figure 6. Blocks for simulating: (a) photocurrent and (b) diode current

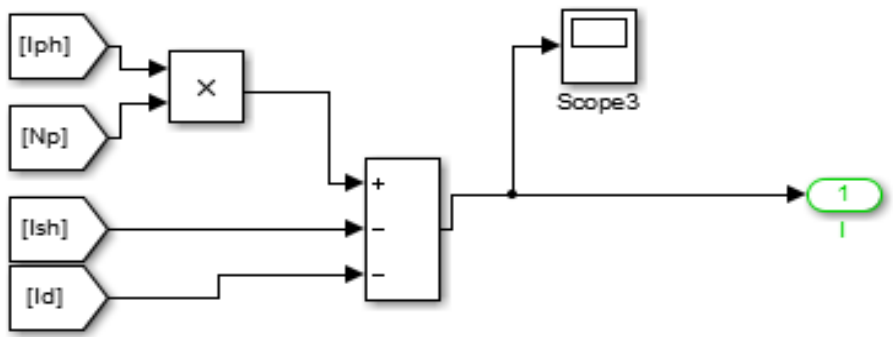

Figure 7. Modeling of panel output current

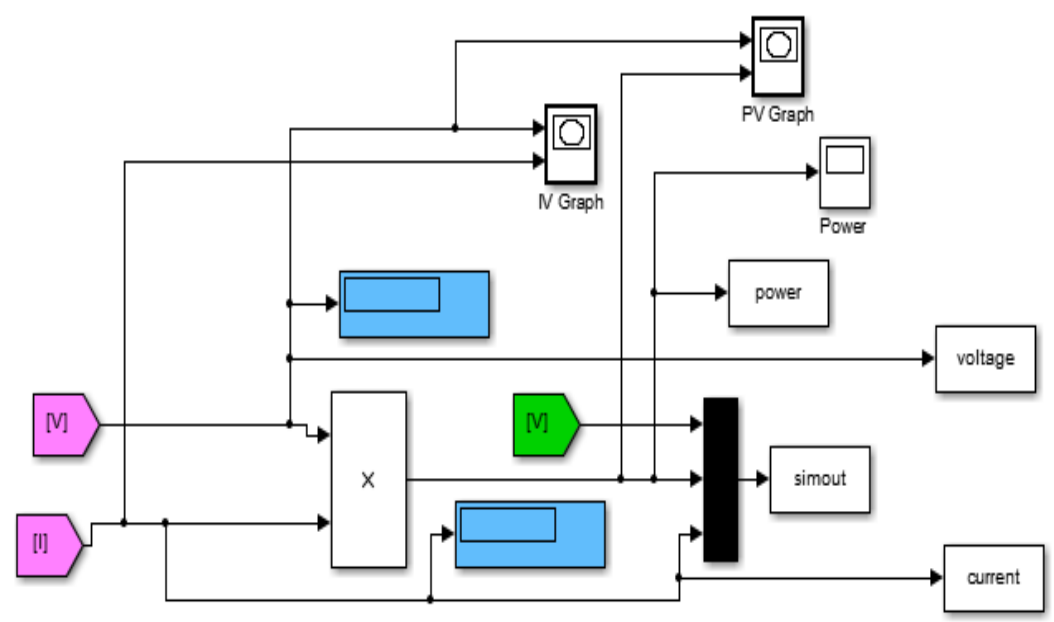

Figure 8. Simulated blocks for estimating power output 


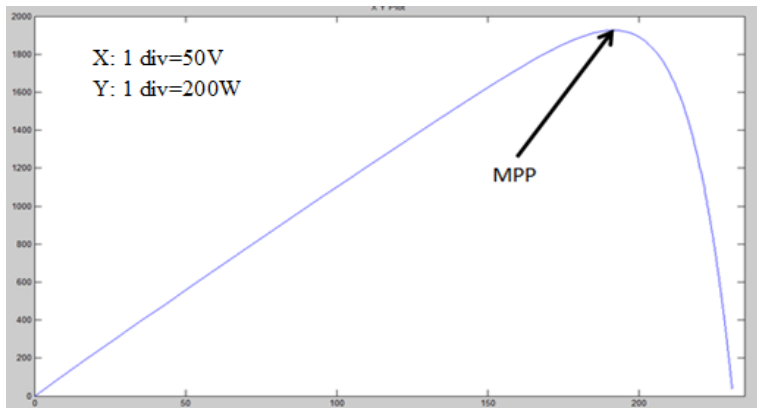

Figure 9. PV characteristic obtained at STC

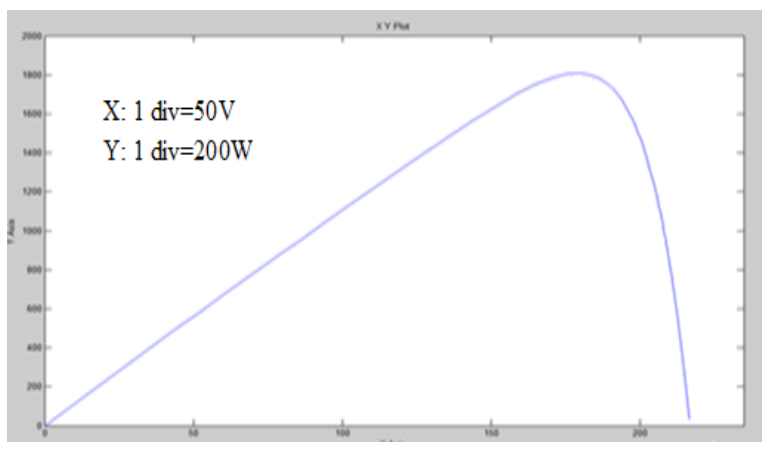

Figure 11. PV characteristic at Temperature of $15^{\circ} \mathrm{C}$ $\left(\mathrm{S}=1000 \mathrm{~W} / \mathrm{m}^{2}\right)$

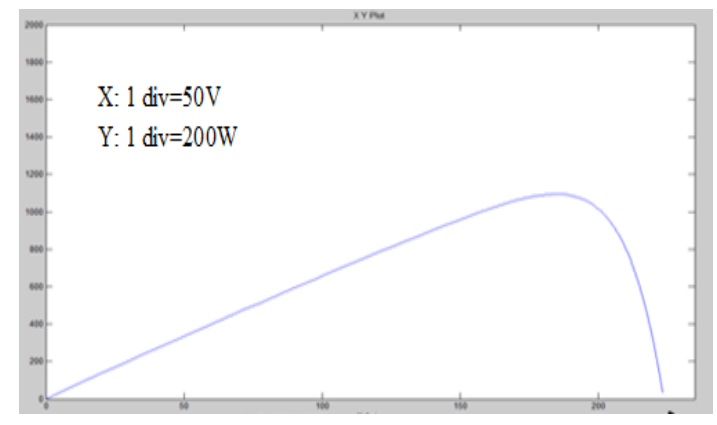

Figure 10. PV characteristic at an insolation of 600 $\mathrm{W} / \mathrm{m}^{2}\left(\mathrm{~T}=25^{\circ} \mathrm{C}\right)$

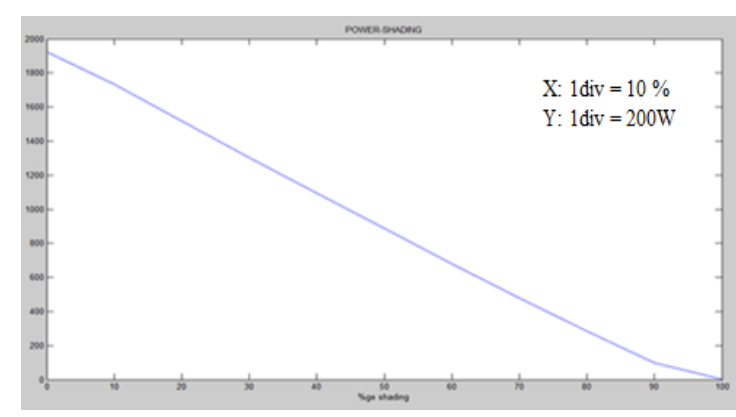

Figure 12. MPP tracked for dynamic \% shading

\subsection{Experimental analysis}

All the simulated results for tracked MPP under dynamic conditions were verified experimentally to validate the effectiveness of MB algorithm. As discussed earlier, variations in the environmental conditions were practically monitored with the help of installed sensors and correspondingly the estimated data i.e variations in current, voltage and power at tracked MPP were recorded through data logger CR 1000. Further, these readings were also monitored in the display panel shown in Figure 5 and stored in computer as well. As done in the case of simulated results, here also we start with first tracking the MPP at STC and the corresponding readings are given in Table 2. Then the experimental results for tracked MPP for dynamic variations in environmental conditions are summarized one by one in Tabular form where apart from the maximum power output, the values of current and voltage readings at MPP have also been specified. The impact of dynamic variation of the radiation intensity on MPPT capability has been investigated for the range of insolation in between $100 \mathrm{~W} / \mathrm{m}^{2}$ to $1000 \mathrm{~W} / \mathrm{m}^{2}$ and the corresponding readings are summarized in Table 3.

Table 2. Experimental PV characteristic at STC

\begin{tabular}{ccc}
\hline Voltage $(\mathrm{V})$ & Current $(\mathrm{A})$ & Power $(\mathrm{W})$ \\
\hline 50 & 11.181 & 584.3 \\
100 & 11.013 & 1124.7 \\
120 & 10.831 & 1298.3 \\
140 & 10.373 & 1558.9 \\
160 & 9.825 & 1780.1 \\
180 & 8.987 & 1882.5 \\
190 & 8.743 & 1924.6 \\
200 & 8.375 & 1895 \\
210 & 8.013 & 1680.8 \\
220 & 6.435 & 1245.6 \\
225 & 3.015 & 795.4 \\
230 & 0.331 & 170 \\
\hline
\end{tabular}


Table 3. MPP tracked for dynamic insolation

\begin{tabular}{cccc}
\hline Insolation $\left(\mathrm{W} / \mathrm{m}^{2}\right)$ & $\mathrm{I}_{\mathrm{m}}(\mathrm{A})$ & $\mathrm{V}_{\mathrm{m}}(\mathrm{V})$ & $\mathrm{MPP}(\mathrm{W})$ \\
\hline 100 & 1.401 & 159.2 & 107.62 \\
200 & 2.729 & 163.4 & 267.32 \\
300 & 3.413 & 171.3 & 459.13 \\
400 & 4.345 & 179.5 & 675.39 \\
500 & 5.136 & 181.7 & 838.12 \\
600 & 6.711 & 185.1 & 1088.31 \\
700 & 7.326 & 187.5 & 1314.52 \\
800 & 9.432 & 189.4 & 1514.81 \\
900 & 10.759 & 190.7 & 1714.37 \\
1000 & 11.103 & 191.5 & 1908.68 \\
\hline
\end{tabular}

Similarly, the MB algorithm is tested for most typical operating condition where partial shading conditions are rapidly varied. It is found that the MB algorithm can effectively track MPP for rapid variations of shading also for which the tracked values are summarized below in Table 4. Here, current also varies significantly and even becomes negative for fully shaded panels because of reverse saturation.

Table 4. MPP tracked for dynamic partial shading

\begin{tabular}{cccc}
\hline Shading $(\%)$ & MPP $(\mathrm{W})$ & $\mathrm{V}_{\mathrm{m}}(\mathrm{V})$ & $\mathrm{I}_{\mathrm{m}}(\mathrm{A})$ \\
\hline 0 & 1923.96 & 229.89 & 11.18 \\
10 & 1731.61 & 229.35 & 10.03 \\
20 & 1517.12 & 225.16 & 9.15 \\
30 & 1304.23 & 221.87 & 7.97 \\
40 & 1093.78 & 213.02 & 6.83 \\
50 & 885.75 & 216.29 & 5.69 \\
60 & 680.47 & 211.34 & 4.55 \\
70 & 479.57 & 204.13 & 3.41 \\
80 & 285.45 & 189.5 & 2.27 \\
90 & 97.72 & 123.29 & 1.13 \\
100 & 0.29 & 0 & -0.17 \\
\hline \multicolumn{4}{c}{} \\
\hline
\end{tabular}

The experimentally measured readings for tracked MPP are then finally compared one by one with those predicted by the simulated model of proposed technique by plotting them on same graphs. Clearly, the results show a very close resemblance between measured and predicted behaviors of proposed MPPT technique and thus validates its effectiveness under dynamic conditions. First the comparison of power output characteristics obtained at STC is shown in Figure 13. It can be seen that MB algorithm is not only capable of tracking MPP in dynamic conditions but at the same time it also gives out a high tracking efficiency. Then the comparative result of MPP tracked for dynamic variations in the insolation has been plotted in Figure 14 and finally results are compared and plotted for the most typical one i.e partial shading.case.

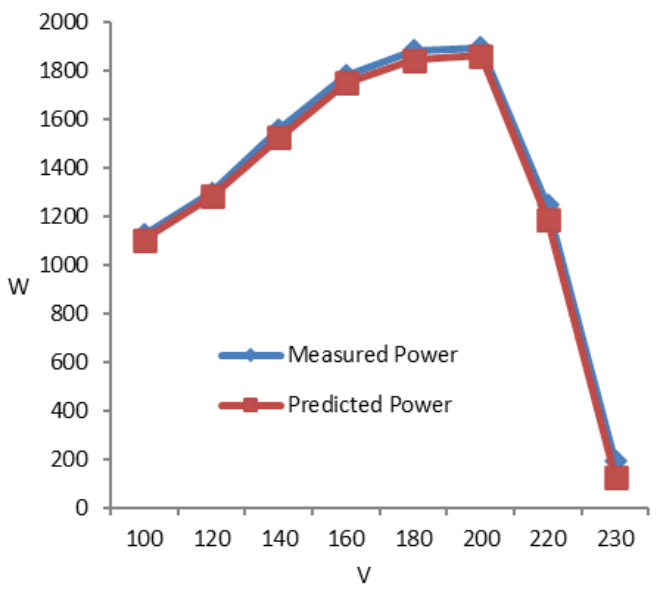

Figure 13. PV power output tracked at STC

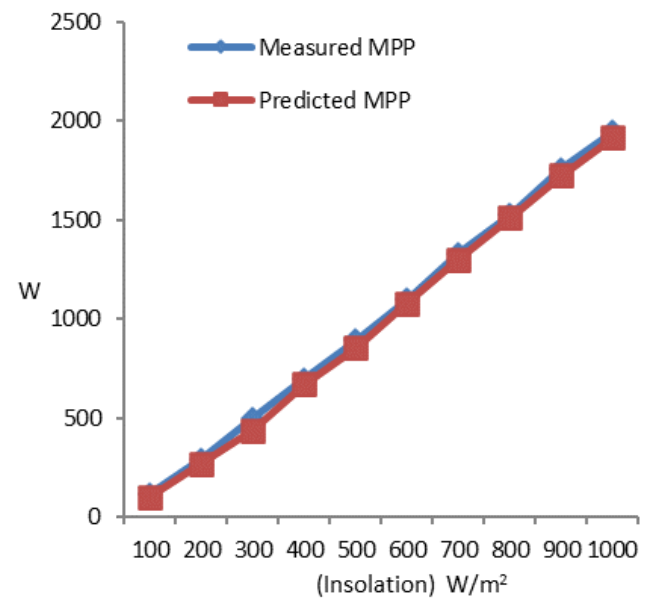

Figure 14. MPP tracked for dynamic insolation 


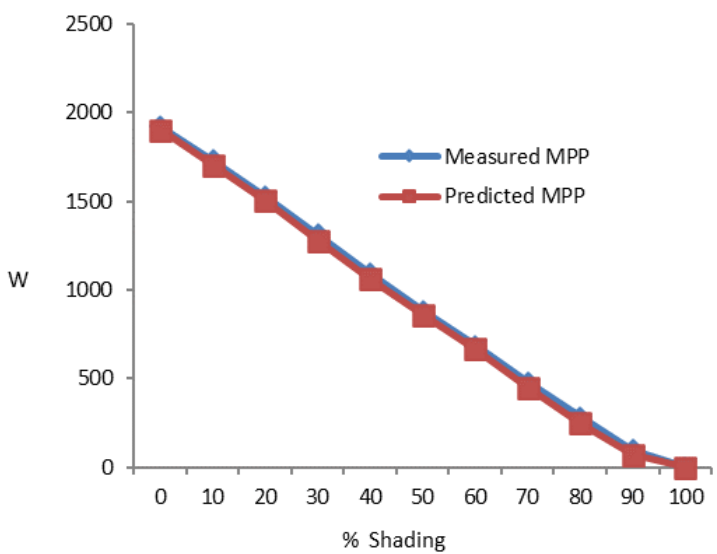

Figure 15. MPP tracked for dynamic shading

\section{CONCLUSION}

With the growing penetration of PV power in distribution system, several challenges are also being faced. One of the major tasks is to ensure that PV panels operate at the optimum point irrespective of environmental variations and hence it requires effective and efficient tracking algorithm. This work has presented an enhanced model based MPPT algorithm which is found to be very effective under dynamic conditions. Here, accurate modeling of PV panel combined with precised estimation of parameters have given a distinct feature to proposed $\mathrm{MB}$ algorithm and thus provided a comprehensive solution to overcome the limitations of earlier techniques. A detailed performance analysis has been carried out to signify the effectiveness of MB algorithm. First simulated results have been obtained which are then investigated experimentally by tracking the real time values. Finally both the results are compared and found to be matching closely. Thus it has been verified that MB algorithm works effectively not only in dynamic conditions where insolation or temperature varies rapidly and continuously, but it has additional capability of tracking the MPP accurately in partial shading conditions also.

\section{REFERENCES}

[1] D. Delgado, M. Carvalho and R. Chacartegui, "Photovoltaic Solar Energy in the economic optimization of energy supply and conversion," IET Renewable Power Generation, vol. 12, no. 11, pp. 1263-1268, Aug. 2018, doi: 10.1049/iet-rpg.2018.0090.

[2] K. Ding, X. Bian, H. Liu and T. Peng, "A MATLAB-Simulink-Based PV Module Model and Its Application Under Conditions of Nonuniform Irradiance," in IEEE Transactions on Energy Conversion, vol. 27, no. 4, pp. 864-872, Dec. 2012, doi: 10.1109/TEC.2012.2216529.

[3] S. K. Kollimalla and M. K. Mishra, "A Novel Adaptive P\&O MPPT Algorithm Considering Sudden Changes in the Irradiance," in IEEE Transactions on Energy Conversion, vol. 29, no. 3, pp. 602-610, Sept. 2014, doi: 10.1109/TEC.2014.232093.

[4] D. Verma, S. Nema, A.M. Shandilya and S. K. Dash, "Maximum power point tracking (MPPT) techniques: Recapitulation in solar photovoltaic systems," Renewable and Sustainable Energy Reviews, vol. 54, pp. 1018-1034, Feb. 2016, doi: 10.1016/j.rser.2015.10.068.

[5] D. Quan, M. Mao, P. Duan, and B. Hu, "An intelligent algorithm for maximum power point tracking in photovoltaic system under partial shading conditions," Transactions of the Institute of Measurement and Control, vol. 39, no. 2, pp. 244-256, Mar. 2015, doi: 10.1177/0142331215606514.

[6] M. Rakhshan, N. Vafamand, M. Khooban and F. Blaabjerg, "Maximum Power Point Tracking Control of Photovoltaic Systems: A Polynomial Fuzzy Model-Based Approach," in IEEE Journal of Emerging and Selected Topics in Power Electronics, vol. 6, no. 1, pp. 292-299, March 2018, doi: 10.1109/JESTPE.2017.2708815.

[7] S. Li, "Linear equivalent models at the maximum power point based on variable weather parameters for photovoltaic cell," Applied Energy, vol. 182, pp. 94-104, 2016, doi: 10.1016/j.apenergy.2016.08.097.

[8] T. H. Kawan and X. Wu, "Maximum power point tracking using a variable antecedent fuzzy logic controller," Solar Energy, vol. 137, pp. 189-200, 2016, doi: 10.1016/j.solener.2016.08.008.

[9] A. Mohapatra, B. Nayak and C. Saiprakash, "Adaptive Perturb \& Observe MPPT for PV System with Experimental Validation," 2019 IEEE International Conference on Sustainable Energy Technologies and Systems (ICSETS), 2019, pp. 257-261, doi: 10.1109/ICSETS.2019.8744819.

[10] S. Marhraoui, A. Abbou, N. El Hichami, S. E. Rhaili and M. R. Tur, "Grid-Connected PV Using Sliding Mode Based on Incremental Conductance MPPT and VSC," 2019 8th International Conference on Renewable Energy Research and Applications (ICRERA), 2019, pp. 516-520, doi: 10.1109/ICRERA47325.2019.8996621. 
[11] S. Thakran, J. Singh, R. Garg and P. Mahajan, "Implementation of P\&O Algorithm for MPPT in SPV System," 2018 International Conference on Power Energy, Environment and Intelligent Control (PEEIC), 2018, pp. 242245, doi: 10.1109/PEEIC.2018.8665588.

[12] J. Kim and H. Cha, "A Novel Variable Step sized MPPT Control based on P\&O Method for Photovoltaic System," 2019 22nd International Conference on Electrical Machines and Systems (ICEMS), 2019, pp. 1-5, doi: 10.1109/ICEMS.2019.8921571.

[13] G. Dhaouadi, O. Djamel, S. Youcef and C. Salah, "Implementation of Incremental Conductance Based MPPT Algorithm for Photovoltaic System," 2019 4th International Conference on Power Electronics and their Applications (ICPEA), 2019, pp. 1-5, doi: 10.1109/ICPEA1.2019.8911186.

[14] U. Chauhan, A. Rani, V. singh and B. Kumar, "A Modified Incremental Conductance Maximum Power Point Technique for Standalone PV System," 2020 7th International Conference on Signal Processing and Integrated Networks (SPIN), 2020, pp. 61-64, doi: 10.1109/SPIN48934.2020.9071156.

[15] H. Rezk, M. Aly, M. Al-Dhaifallah and M. Shoyama, "Design and Hardware Implementation of New Adaptive Fuzzy Logic-Based MPPT Control Method for Photovoltaic Applications," in IEEE Access, vol. 7, pp. 106427106438, 2019, doi: 10.1109/ACCESS.2019.2932694.

[16] M. Nour Ali, "Improved Design of Artificial Neural Network for MPPT of Grid-Connected PV Systems," 2018 Twentieth International Middle East Power Systems Conference (MEPCON), 2018, pp. 97-102, doi: 10.1109/MEPCON.2018.8635202.

[17] C. Lee, H. Tsou, T. Chou and K. Weng, "Application of the hybrid taguchi genetic algorithm to maximum power point tracking of photovoltaic system," 2018 IEEE International Conference on Applied System Invention (ICASI), 2018, pp. 231-234, doi: 10.1109/ICASI.2018.8394575.

[18] O. Kırcioğlu, M. Ünlü and S. Çamur, "The PSO Based Global Maximum Power Point Tracker," 2019 11th International Conference on Electronics, Computers and Artificial Intelligence (ECAI), 2019, pp. 1-4, doi: 10.1109/ECAI46879.2019.9041926.

[19] J. A. Nemours and S. Chowdhury, "Performance Analysis of Fuzzy Logic Maximum Power Point Tracking Scheme for Solar PV System Under Varying Load and Atmospheric Conditions," 2019 IEEE PES/IAS PowerAfrica, 2019, pp. 134-139, doi: 10.1109/PowerAfrica.2019.8928895.

[20] K. Bataineh, "Improved hybrid algorithms-based MPPT algorithm for PV system operating under severe weather conditions," IET Power Electronics, vol. 12, no. 4, pp. 703-711, 2019, doi: 10.1049/iet-pel.2018.5651.

[21] I. Abadi, C. Imron, Mardlijah, R. D. Noriyati, "Implementation of Maximum Power Point Tracking (MPPT) Technique on Solar Tracking System Based on Adaptive Neuro-Fuzzy Inference System (ANFIS)," E3S Web of Conferences, vol. 43, p. 01014, 2018, doi: 10.1051/e3sconf/20184301014.

[22] A. Beltran, C. Hughes and S. Das, "Improved Maximum Power Point Tracking of Partially Shaded PV Arrays Using Particle Swarm Optimization with Zone Initialization," 2019 IEEE 46th Photovoltaic Specialists Conference (PVSC), 2019, pp. 0663-0667, doi: 10.1109/PVSC40753.2019.8980469.

[23] K. Lappalainen and S. Valkealahti, "Fluctuation of PV Array Global Maximum Power Point Voltage During Irradiance Transitions caused by Clouds," IET Renewable Power Generation, vol. 13, no. 15, pp. 2864-2870, 2019, doi: 10.1049/iet-rpg.2019.0085.

[24] A. Al Mansur and M. R. Amin, "Performance Investigation of Different PV Array Configurations at Partial Shading Condition for Maximum Power Output," 2019 International Conference on Sustainable Technologies for Industry 4.0 (STI), 2019, pp. 1-5, doi: 10.1109/STI47673.2019.9067998.

[25] B. C. Babu and S. Gurjar, "A Novel Simplified Two-Diode Model of Photovoltaic (PV) Module," in IEEE Journal of Photovoltaics, vol. 4, no. 4, pp. 1156-1161, July 2014, doi: 10.1109/JPHOTOV.2014.2316371.

[26] H. K. Mehta, H. Warke, K. Kukadiya and A. K. Panchal, "Accurate Expressions for Single-Diode-Model Solar Cell Parameterization," in IEEE Journal of Photovoltaics, vol. 9, no. 3, pp. 803-810, May 2019, doi: 10.1109/JPHOTOV.2019.2896264.

[27] A. Ortiz-Conde, D. Lugo-Muñoz and F. J. García-Sánchez, "An Explicit Multiexponential Model as an Alternative to Traditional Solar Cell Models with Series and Shunt Resistances," in IEEE Journal of Photovoltaics, vol. 2, no. 3, pp. 261-268, July 2012, doi: 10.1109/JPHOTOV.2012.2190265. 